\title{
CORRESPONDENCE
}

\section{Malaria vaccines?}

SiR - Considerable effort is being directed towards producing a practical and effective vaccine against the most malignant human malaria, that caused by Plasmodium falciparum. The belief that this is feasible was based on the realization that human malaria induces effective specific immune responses which are protective. The best characterized of these are antibody-mediated, directed against stage and species specific antigens of sporozoites and merozoites and seem to act by preventing the entry of these two extracellular forms into their respective target cells, hepatocytes and erythrocytes.

However, the clinical effectiveness of natural immunity in exposed subjects is partially offset by processes generated by living plasmodia which permit evasion of immune effector mechanisms. These include the bewildering degree of antigenic diversity generated by the parasite as it differentiates through successive stages; also its ability to compromise host immunity through induction of immunosuppression and through polyclonal activation of lymphocytes and probably also by production of soluble antigen.

Experimental vaccination with, for example, irradiated sporozoites or non-invasive merozoites, induces more effective immunity than does natural infection, indicating that nonviable forms of the parasite can stimulate protective immunity without activating processes favouring parasite survival.

The logical step from these observations is to develop stage-specific vaccines based on sporozoites and merozoites. The difficulty of obtaining these forms in sufficient quantities for even a restricted trial of adequately standardized material has encouraged detailed investigations into the structure of plasmodial antigens in the hope of identifying those involved in inducing protective immunity. This task was beset with technical difficulties, but the prospects have been transformed by the advent of techniques of somatic cell hybridization to generate cell lines which secrete monoclonal antibodies of defined specificity. The very first clone of antibody secreting cells raised against sporozoites led directly to the isolation of a stage-specific, surface-coat component of molecular weight 44,000 with the potential for promoting immunity to this clinically important stage of infection. With these specific probes it is becoming feasible to attempt the in vitro production through DNA cloning of plasmodial antigens having putative protective activity.

It is not surprising that such work should attract the attention of commercial interests, not least from those most recently founded and best equipped to exploit its potential. This intervention gives promise of greatly strengthening the malaria vaccine research effort, but some of its consequences may soon retard progress. Work on a malaria vaccine is unusual in requiring access to materials and facilities so diverse as to demand the collaboration of many laboratories. These requirements include a wide range of adequately characterized and geographically remote plasmodial species, their in vitro culture, the use of breeding colonies of appropriate mosquito vectors, access to those few primate species susceptible to human malaria and eventually the organization of adequately controlled and locally acceptable clinical trials in countries which may have only rudimentary public health facilities. Despite these requirements some institutions are contemplating individually beneficial patent rights on segments of the work. This action already threatens to disrupt in part the complex web of collaborative work so central to success.

Malaria menaces over a third of the world's population and is confined almost exclusively to developing countries. There are around 150 million cases per year and over a million African children die annually of the disease. A recent intensive survey has shown that in the vast African savannah no known method of prophylaxis, however thoroughly dispensed, can in practice be effective. Elsewhere, for example in India and Sri-Lanka, it has proved impossible to sustain prophylactic measures and malaria is alarmingly resurgent. The cost of a developed malaria vaccine can never be recouped from the relatively impoverished countries which require it most urgently. Its manufacture and distribution will inevitably require massive subsidy from international governmental and charitable funds. Under these circumstances can any institution justify a claim to benefit individually from a restricted aspect of work, however important, which contributes to the development of a malaria vaccine? Procedural agreement is urgently required in this area of research. Representatives of major organizations involved should meet together forthwith under appropriate international auspices and agree a moratorium on all patent rights relating to the development of a human malaria vaccine. Is it really naïve to believe that this enterprise cannot be sustained solely on the basis of its scientific feasibility and enormous practical potential for the health of mankind?

S. COHEN

Department of Chemical Pathology,

Guy's Hospital Medical School,

London SE1, UK

\section{Nobel Prize rules}

SIR - Your editorial on changing the Nobel Prize rules (Nature 23 October, p.667) is right on all points. However, it does not mention one vital question - the statistics of sampling.

One way that the Nobel Foundation uses to select candidates is to write at random to about 50 universities for proposals. This list is different each year and comprises a sample of the about 900 universities around the globe (Encyclopaedia Britannica, Micropaedia X, pp.888-1007). As most of these universities are likely to have a chair in nuclear physics, but only about $10 \%$ one in spectroscopy, less than $10 \%$ in flow phenomena (hydro- and aerodynamics ...); less than $5 \%$ atmospheric physics; less than $2 \%$ aerosol physics etc., the odds that a nuclear physicist will get the letter and nominate somebody within his own field, are incomparably greater than for any other branch of physics.

Proof: Between 1950 and 1973, 16 Nobel prizes went to some aspect of nuclear physics, 3 were awarded for low-temperature phenomena; 3 for optics; 2 for quantum electrodynamics and 1 each for magnetism and semiconductors. Probably similar biasses exist in chemistry and medicine.

In conclusion: the Nobel Prize goes by preference to the disciplines with the largest number of scientists. In other words, it is biassed towards specialities best endowed with funds. This means that governmental budgeting interacts with the award.

It is surprising that such a select scientific body should not be aware of such a basic sampling error. More than a sampling error; this "pseudorandomization"' goes against Nobel's will. At the end of the nineteenth century, physics was comprehensive and subdivision in specialities almost unheard of. Since then, electronics, atmospheric physics, rocketry, astrophysics and many, many other branches have become so detached, that scientists working in such areas are almost excluded from being considered for the prize.

M. BENARIE

\section{Institut National de Recherche \\ Chimique Appliquée,}

Brétigny, France

\section{Fungal food}

SIR - The recent article on fungal foods (Nature 4 September, p.6) states that the protein gap that was believed to exist in many developing countries has now been declared a myth. 1 have discussed a similar problem with Mr J. Todd, Executive Assistant to the Unitarian Service Committee of Canada - a Canadian charitable organization engaged in overseas relief and development in cooperation with governments of the leastdeveloped countries. These countries are specifically chosen on the basis of a per capita income being less than $\$ 200$ per year. The agency is working in six such countries.

Mr Todd's description of the protein shortages in the many communities in which he works and the desperate need for protein in a wide variety of forms contrasts sharply with your statement. Although such countries are probably unable to buy protein from overseas, the lack of demand based on such inability can surely not be taken as an absence of need.

P.J. HEALD

Memorial University of Newfoundland, St John's, Newfoundland, Canada AIB $3 X 7$

\section{AChE uses}

SIR - What, pray, is wrong with the determination of any government to attempt to protect its citizens, be they either soldiers or civilians, against the use of "nerve gas" (?organophosphorus) weapons? How can this be thought of as "distortion of technology"? R.G. Elles et al. (Nature 9 October, p.480) are adopting a stereotyped "holier than thou" attitude more appropriate to the behaviour of our "Praise-the-Lord Barebones" forebears of the post-Civil War period - "smelling-out" evils when they would be better occupied in haranguing protest meetings of a nonscientific nature; such letters are inappropriate for a science-based Nature.

R.A. Davis

Rodent Research Department, Tolworth Laboratory, Tolworth, UK 